\title{
A question of biology
}

\section{Complete genome sequences have provided a plethora of potential drug targets. But the hard task of finding their weak spots is just beginning, as Caitlin Smith finds out.}

W ith the complete sequencing of the human genome, one might think that the identification of new potential drug targets would be coming to a halt. Yet there are still many exciting developments to come in the field of target identification, with technological advances enabling challenging biological questions to be addressed in increasingly creative ways.

According to Kevin Fitzgerald, group leader of applied genomics at Bristol-Myers Squibb headquartered in New York, one of the biggest challenges is to understand the underlying physiology of drug targets. "We do not yet understand all of the basic underlying principles and rules," he says. "We are left trying to interpret and predict the behaviour of dynamic processes based on small snapshots in time. The electrifying part of being involved in biology today is that the technology is beginning to catch up. If you are able to take enough snapshots under different stimuli and conditions, over time, concepts and rules do emerge."

Part of this challenge is to identify interactions between drug targets, which are generally proteins such as receptors and enzymes, and the proteins and other molecules that regulate them. As Jan Mous, president and chief executive of IntegraGen of Evry, France, says: "The most exciting development is also the most challenging: to first understand and appreciate the subtle interaction of many proteins and different messengers and hormones steering the function of our cells and organs, and then pick the best target for drug interaction giving the most favourable ratio of wanted versus unwanted effects."

Interactions are often subtle and complex, requiring a multidisciplinary approach and the use of informatics systems to make sense of the increasingly complicated data. "A very tight integration of computer sciences and mathematics with experimental biology and chemistry has to be the modus operandi for the future drugdiscoverybusiness," says Mous.

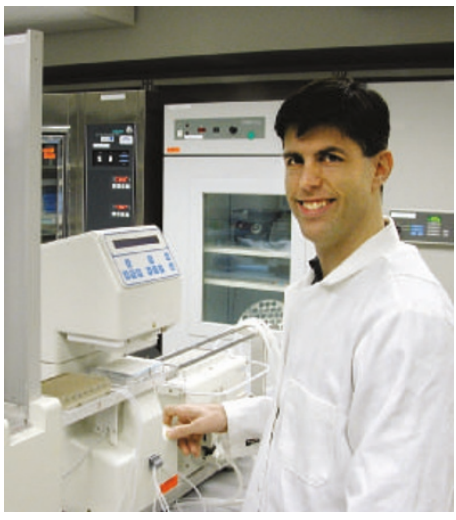

Kevin Fitzgerald: finding rules.
One approach to investigating physiology is through genomics. Now that the human, mouse and rat genomes have been sequenced, high-throughput genomics can come into its own. Ingenium Pharmaceuticals in Martinsried, Germany, uses largescale chemical mutagenesis and screening to look for novel, medically relevant biological mechanisms in mouse and rat mutants. When a phenotype of interest is found, the mutation is located by high-speed positional cloning and a line of model animals can be produced. Further biological information can be gathered through geneexpression and cell-pathway analysis, complementing the pathophysiology observed in the mutant animal.

A new mechanism of sensitization to the hormones leptin and insulin was recently discovered using this technology. "The effect

\section{RUNNING INTERFERENCE ON THE GENOME}

RNA interference (RNAi) is a powerful method of gene silencing using double-stranded RNA, which is converted to small inhibitory RNA (siRNA) as the active agent. siRNAs are now hot property as reagents for knocking down gene expression in target identification and validation assays, and as possible therapeutics for silencing genes associated with disease.

According to Christophe Echeverri, chief executive and scientific officer of Cenix BioScience in Dresden, Germany,

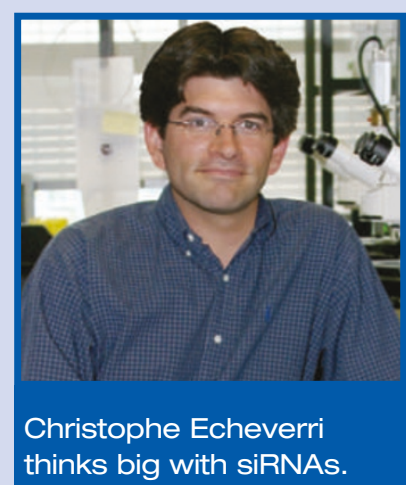

high-throughput RNAi is the most exciting recent development in target discovery and validation. "The key advance here is the development of genome-wide libraries of siRNAs to enable genome-wide screens in human cells," he says. "It offers the most direct, cost-efficient way to get from gene sequence to gene function at a genomic scale." As part of its drug-discovery and target-validation programmes, Cenix has designed siRNAs against more than $98 \%$ of the human, mouse and rat genes identified by the genome-sequencing projects; the siRNAs themselves, for all these genes listed in the RefSeq database, are available to order through Ambion of Austin, Texas, which also offers an off-the-shelf library of nearly 600 kinase siRNAs made to the Cenix designs.

The siRNA drug-development company Atugen in Berlin, Germany, also develops siRNAs as both reagents for target identification and validation and as potential therapeutics. Atugen's technology platform for target identification uses various messenger RNA knockdown techniques, including proprietary siRNAs chemically modified for increased stability, and a proprietary antisense method called Geneblocs. "These are used in combination with proprietary liposomal formulations to elucidate gene function in specialized cellular assays and animal models," says Klaus Giese, chief scientific officer at Atugen. "Matching results found with siRNA and Geneblocs will substantially increase the probability that an observed phenotype is clinically relevant."

RNAi allows more questions to be asked of mammalian cells than ever before. "The ability of RNAi to allow the 'yeastification' of mammalian cells in terms of genetics is very exciting," says Kevin Fitzgerald, group leader of applied genomics at Bristol-Myers Squibb in New York. "With RNAi we can now begin to approach the genetics of tumour-suppressor genes in a whole new way. We have known that the loss of these genes is very important in the development of cancer for years, but we have been unable to develop specific drugs directed at them. With RNAi we can now ask whether there are any druggable targets in the genome that will lead to the death of cells missing tumour suppressors but leave normal cells alone. This is a question that in mammalian cells we simply could not have asked a few years ago." 
of the inhibition of the newly discovered pathway is that our mouse model has highly improved tolerance to glucose while requiring only a fraction of insulin for this regulation," says Ingenium's chief executive Michael Nehls. "In contrast to other insulinsensitizing effects, the mice do not become obese - on the contrary, they slim under a high-fat diet. We believe this could be a breakthrough in obesity/diabetes research," says Klaus Dembowsky, vice-president of drug discovery

\section{From genotype to phenotype}

Despite the increasing emphasis on proteomics in target identification, DNA microarray technology is still a powerful technique for identifying genes involved in susceptibility to diseases. Target discovery and identification should soon be benefiting from the DNA microarrays of the complete human genome now sold by several companies. First to market in July 2003 was NimbleGen in Madison, Wisconsin, with a chip containing 200,000 probes, with an average of five probes per gene. This was followed in October 2003 by the Human Genome U133 Plus 2.0 array from Affymetrix in Santa Clara, California, with 1.3 million probes and the ability to analyse the expression of about 47,000 different transcripts. The most recent human wholegenome chip, launched last month, comes from Agilent Technologies, in Palo Alto, California. Agilent's double-density-format chip represents about 41,000 genes and, with Agilent's ImaGene image-analysis software, is compatible with most commercial microarray scanners for $25 \times 75$-mm chips.

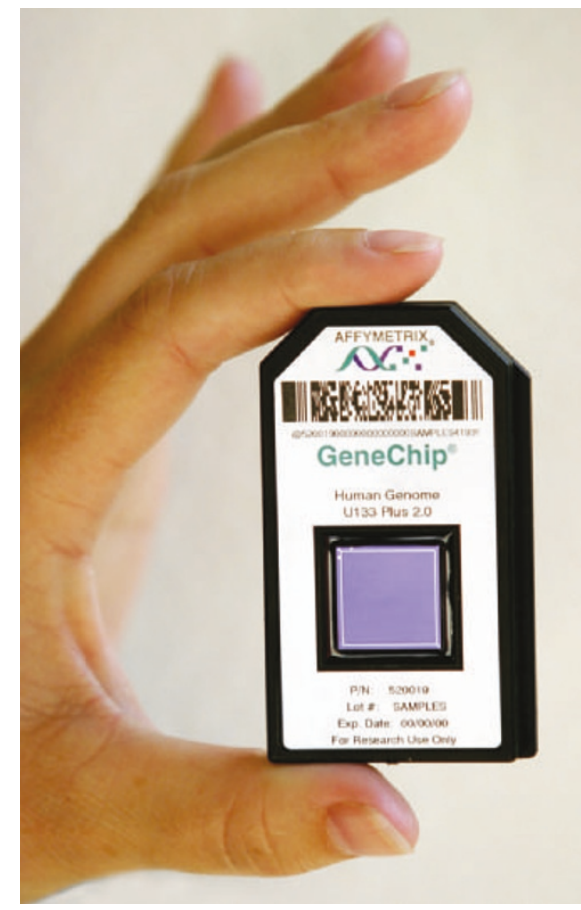

What it means to be human?

This open-platform approach, the company claims, will make it easier for scientists to move from their own homemade chips to the Agilent chip.

Microarrays are already widely used to study gene expression and to detect sequence features such as single-nucleotide polymorphisms (see Nature 422, 917-923; 2003). Future uses will include assays to study the many forms that a protein target may take as a result of alternative splicing. "For RNA analysis, over the next year, we will start to see assays for efficient splice variant analysis that will enable us to move from gene-specific analysis to transcript-specific analysis," says David Craford, vice-president of business development at Affymetrix. "More data are emerging to suggest that for many diseases this will be incredibly useful."

According to Greg Yap, senior marketing director in DNA analysis at Affymetrix, one of the newest and most exciting applications of microarrays to target identification is the possibility of genome-wide association studies of complex genetic diseases. "The experiments that geneticists really want to do are to find genes that cause complex diseases in unrelated clinical populations," says Yap. "Finding genes that cause a disease in a family makes a nice story, but finding genes causing a disease in the world makes a drug target." Studying genetically unrelated people is typically difficult, owing to the numbers of genetic markers per person that need to be studied. The advent of microarrays has made individual genotyping feasible, "making it realistic to compare the genomes of people with and without a disease at high-enough resolution to find potential drug targets", he says.

Pharmaceutical company Roche in Basel, Switzerland, is using the Affymetrix wholegenome expression arrays to identify genes that play key roles in disease pathways. "One approach for new target discovery that we use is to identify the genetic basis for disease susceptibility using a coupled genetic and genomic analysis," says Gary Peltz, head of genetics and genomics at Roche. "Having the complete genomes of several organisms (human, mouse and rat) available on chips is a major advance. It allows complex biology,

\section{DRUG DISCOVERY IN REVERSE}

Reversing the current drug-development path of 'target to drug to phenotype', chemogenomics, or chemical genomics, starts with a known drug that causes an interesting disease-relevant phenotype in vitro or in vivo, and then identifies the cellular target(s) for that drug. Chemogenomics is a powerful tool in target identification because you start with an active small molecule. By definition, any targets found are 'druggable' and are likely to be significant in the phenotype caused by the drug.

Seth Cohen, who recently moved from Millennium Pharmaceuticals to Caliper Life Sciences in Mountain View, California, likens chemogenomics to "reverse drug discovery". In other words, you take a drug compound with known effect and analyse in detail the result of exposure of the cell or organism to the compound. "This allows for a more precise understanding of the mechanism of action as well as potential causes of side effects. It also enables more intelligent subsequent screening of compounds with better, more relevant assay readouts," he says.

Iconix Pharmaceuticals in Mountain View, in collaboration with MDS Pharma Services of Lincoln, Nebraska, applies chemogenomics to the study of comprehensive responses of rats to treatment by a compound. Iconix uses CodeLink microarrays from Amersham Bioscience in Piscataway, New Jersey, which carry 10,000 probes for rat genes and expressed sequence tags, to measure gene expression in tissues from rats that have been treated with different drugs. MDS and Iconix have identified more than 250 clusters of genes whose expression changes in response to 600 drugs. "These clusters of genes, or 'drug signatures', describe and predict many specific mechanisms of action of the drug in the whole animal without the caveats of in vitro systems," says Pauline Gee, vice-president of predictive biology at MDS. Iconix's DrugMatrix database now includes more than 200 of these drug signatures, which are used by drug companies such as Bristol-Myers Squibb to help select candidate drug molecules more efficiently and to improve their understanding of the safety of new compounds before preclinical development. 
especially as it relates to disease processes, to be explored in an efficient manner." Using a mouse model of a trait associated with a human disease, his group identifies regions of the mouse chromosome that control susceptibility to the disease-associated trait. Gene-expression profiling with the mouse microarrays is also done on target tissues from the mouse strains under study.

"The differentially expressed genes encoded within the identified chromosomal regions are the candidate genetic susceptibility loci," says Peltz. "We then analyse the function of the candidate genes (and pathways) in the biology related to the disease." A mouse model of osteoporosis was recently developed using this method, and an enzyme identified that affects bone mass and bone quality, which led to the development of a new potential therapeutic for treating osteoporosis.

IntegraGen's GenomeHIP technology platform takes a different microarray-based approach to discovering genes linked to complex disease traits in humans. The technology uses DNA chips for genomic comparisons of pairs of related individuals with the same disease to discover areas in which the genomes are identical — on the assumption that at least one of the relevant disease genes in such individuals will be identical in both. Crucial to IntegraGen's technology is the prior removal of most of the polymorphisms that differentiate the two genomes, cutting down the amount of genome that has to be searched. The company claims that its method is cheaper and faster than conventional methods of gene identification, such as linkage analysis using microsatellite markers. Unlike such methods, which can require several generations of many different families, the GenomeHIP technology can identify potential disease genes with high statistical significance in a relatively small sample of patients (50-150 pairs of related individuals with the same trait) in less than nine months.

Using this platform, IntegraGen recently identified a mutant G-proteincoupled receptor that is strongly associated with obesity in various populations. "The most frequent mutation observed was demonstrated to impair the signalling through this receptor by its natural ligands," says Mous. "The normal function of this receptor, which is predominantly expressed in the gut, is to transmit an anorexic signal, which could be impaired in patients expressing the mutant form."

The advent of the complete human-gene chips is generally welcomed as a important tool. But Fitzgerald cautions that, like most molecular tools, gene chips have limitations of which one must be aware. "Experimental design with an eye towards rigorous statistical analysis is crucial," he says. "Gene chips produce a large number of individual gene patterns and if one is asking a computer program to find patterns in the data, it will find patterns. If the experiments were not correctly designed and controlled, those patterns can be quite misleading."

$\mathrm{He}$ also stresses the importance of remembering that in general, gene chips measure correlative rather than causative events. Andrea Gnirke of Xantos Biomedicine in Munich, Germany, also makes the point that the chips provide an analytical challenge. "Chip data can only be correlative data and implicate an enormous effort for data analysis, and it is difficult to sort out the 'good' targets that have causative effects in diseases," she says.

And because much cellular regulation occurs at the level of proteins, "increased transcript production as measured by gene chips does not always correlate with production of protein, and even if more protein is produced it may not be active because it requires post-translational modification or relocalization within a cell", cautions Fitzgerald. Jean-Jacques Yarmoff of Hybrigenics agrees: "Modifications of proteins lead to an additional level of complexity that is not necessarily addressable by humangene chips."

\section{Tackling the 'interactome'}

Because interactions between proteins are key to their function, determining the pathways within which a potential drug target

\section{GETTING UP SPEED}

Multiplexing in highly automated systems is one way of speeding up the assays used for target identification. Bio-Rad in Hercules, California, offers Bio-Plex, a multiplex analysis system that allows the simultaneous analysis of up to 100 different biomolecules (proteins, peptides or nucleic acids) in a single microplate well. Bio-Plex integrates assay kits, software, calibration and validation tools, and instrumentation into a complete system. The Bio-

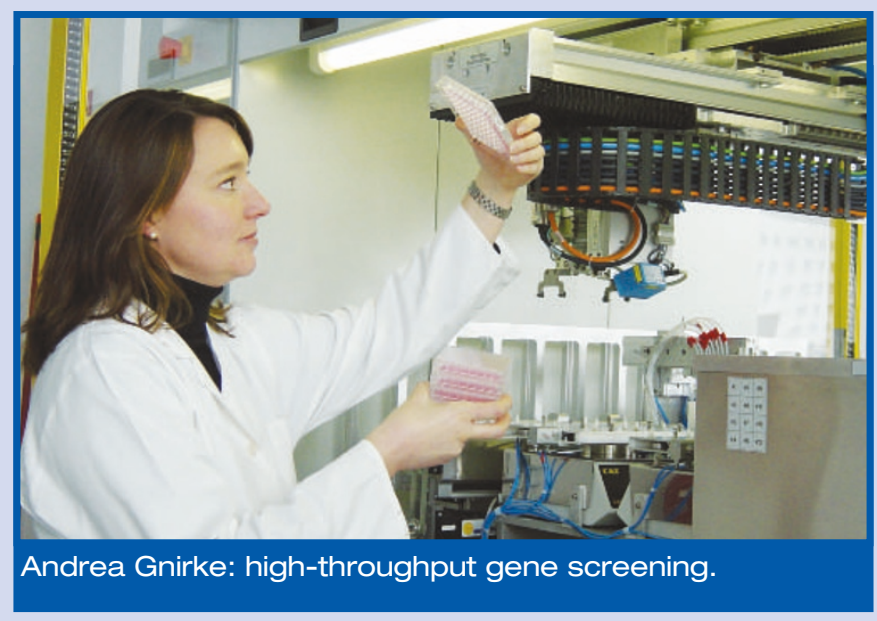

Plex suspension array and workstation uses three main technologies: a family of fluorescence-tagged microspheres from Luminex in Austin, Texas; a flow cytometer that uses two lasers to measure biochemical reactions occurring on the surface of the microspheres; and a high-speed digital signal processor to manage the fluorescent output.

In its target-identification programme, Xantos Biomedicine of Munich, Germany, uses high-throughput cellular screening assays to study gene function in parallel for thousands of genes. For whole-genome screening, its proprietary XantoPrep and XantoScreen high-throughput system screens complementary DNA libraries by overexpression in transient transfection systems, enabling Xantos to carry out functional analysis on up to 100,000 different cDNAs per month. "Genes or proteins are selected by their ability to directly influence physiologically relevant processes or pathways in cellular assays, leading to a very small number of relevant hits compared with expression-profiling studies, for example," says Andrea Gnirke, senior scientist for expression analysis at Xantos. These methods have been used to identify secreted proteins influencing the differentiation of fat cells, which play a major role in the development of insulin resistance, and which Xantos hopes could have potential for the treatment of obesity-related type II diabetes. An interesting future direction for Xantos, along with many other companies, includes RNA interference (RNAi). "Xantos is implementing a confocal high-content imaging screening system and aims to integrate RNAi technology for high-throughput application," says Gnirke. 
acts in the cell and the interactions it makes with other proteins are crucial. To learn more about these complex interactions, researchers are starting to map and study the 'interactome', the comprehensive listing of which protein interacts with which in an organism. A recent breakthrough by Curagen researchers is the production of an interactome for Drosophila melanogaster, the first comprehensive protein-interaction map for a multicellular organism. They used a high-throughput version of the yeast twohybrid method to identify more than 20,000 unique interactions involving 7,000 genes in Drosophila. Interactions within and between protein complexes were also studied using mathematical modelling.

The study points to a connection between proteins known to be altered in human diseases and the 'druggable' classes of enzymes those enzymes known to bind and be altered by small-molecule drugs. This is a relief for those trying to find potential therapies for human diseases such as cancer, heart disease and diabetes. Many of the interactions discovered in the Drosophila study will now be examined further in human cells for their relevance to disease. Curagen has obtained a similar set of human protein-protein interaction data by the same methods, but is keeping them under wraps for now.

In another approach to target discovery, Curagen has developed a group of targets it calls the 'pharmaceutically tractable genome' (PTG). This is a collection of about 8,000 genes whose products are predicted to make good targets as judged by one of several criteria: they are present in blood or on cell surfaces, rendering them accessible to drugs and antibodies; or are in a class of intracellular proteins that can be modulated by small molecules. This approach allows researchers to focus resources on investigating targets that are both novel and yet likely to lead to successful drugs.

Paris-based Hybrigenics also uses functional genomics for target identification, based on a proprietary yeast two-hybrid method that evaluates which proteins interact with the protein of interest, and whether the interaction is statistically significant. Yarmoff, senior director of business development, says that the company's approach also provides functional information. "Because we obtain many fragments which interact with the yeast two-hybrid bait, we can compute the intersection of these fragments to define the selected interacting domain (SID)," he says. "This is extremely useful information, as by comparing the SID with known functional domain sequences, we can understand not only which protein our bait interacts with, but also the function of the domain at which this interaction takes place. The richness of this information is what allows us to go beyond simple target
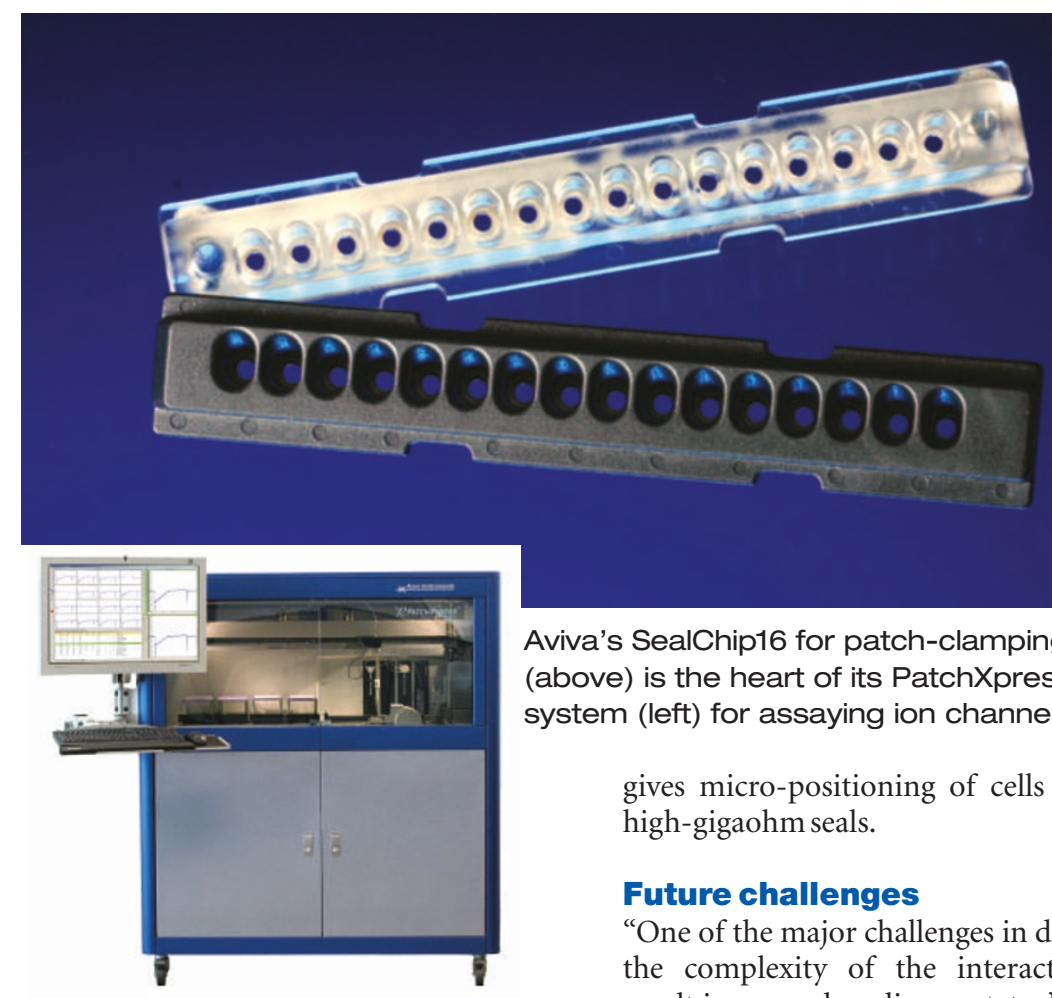

identification to use this information for target validation.” The large amounts of data generated by this approach can be visualized using Hybrigenics' PIMRider viewer, which enables these protein-interaction networks to be explored.

\section{Channel hopping}

Ion channels are the basis of neural function and so are attractive drug targets but, historically, screening for drugs that affect ion channels has been a bottleneck in target identification because the tests cannot be automated successfully - the quality of the data obtained has not been good enough. The alternative, electrophysiological screening of individual cells by a researcher, produces high-quality data, but at a pace that is slow by drug-development standards, and is extremely monotonous and tedious to do. Advances in this area are much needed, because although analysis of the human genome has predicted that ion channels could account for up to a quarter of all potential new targets, only $5 \%$ of drugs currently on the market affect ion channels.

To address this problem, Aviva Biosciences in San Diego, California, has developed its SealChip16 system. This makes high-throughput, high-quality, patchclamp recordings from single cells, in conjunction with Aviva's PatchXpress7000, a high-throughput patch-clamp workstation developed in collaboration with Axon Instruments of Foster City, California. Aviva's system incorporates a high-throughput planar patch-clamp technology that gives micro-positio
high-gigaohm seals.

\section{Future challenges}

"One of the major challenges in discovery is the complexity of the interactions that result in normal or disease states," says Seth Cohen of Caliper Life Sciences in Mountain View, California. "In the past, a simplistic view was, by necessity, taken, which resulted in many drugs failing in preclinical or clinical trials for lack of efficacy or side effects. The newer approach must account for this complexity. The holistic systems-biology approach to research will be necessary to overcome this challenge."

Mous agrees: "A challenging new development in the field of drug-target discovery is systems biology, or the recognition that genes, or better the gene products, are part of, and function, in large complex networks. Understanding the malfunctions in these large pathways will allow the selection of the best drug target for compensating the malfunction of the homeostasis of a biochemical process in a diseased person."

Peltz stresses that studying target pathways will be critical to identifying a target that is important in disease. "The major challenge is the lack of 'connectivity' with pathways for most of the genes in the genome," he says. Studying the disease pathway is the best way to find targets that are most suitable for drug development, he adds. Unfortunately, this is nearly impossible to do at present, because we do not know enough about most genes' interactions. This lack of information, says Peltz, "makes many of the genes we identify as associated with a disease 'isolated islands' that we cannot connect with the 'mainland'. Hopefully, the proteininteraction network maps that are being prepared in various organisms will help to fill this gap."

Caitlin Smith is a science writer based in Portland, Oregon. 


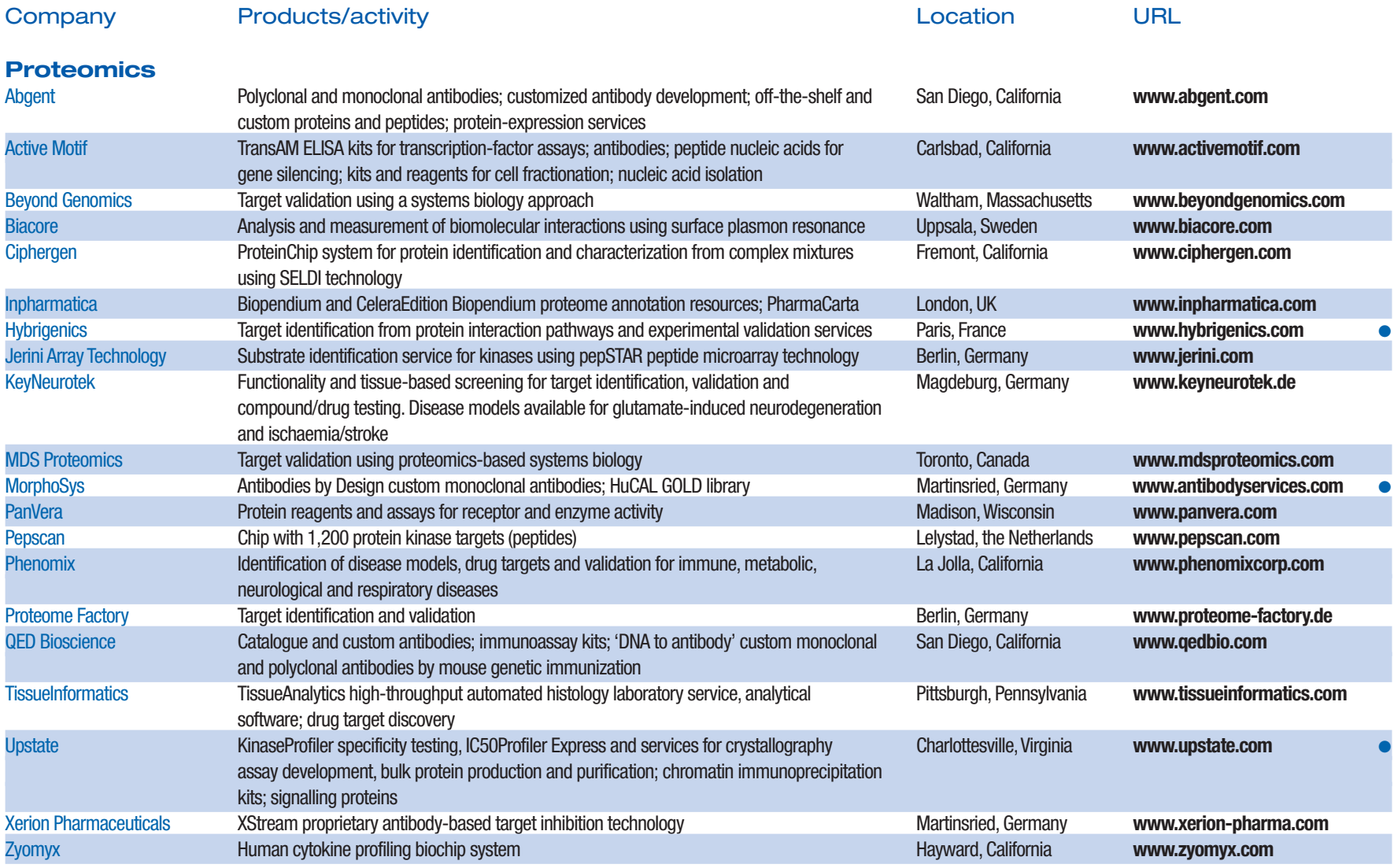

\section{Genomics and gene-expression profiling}

Aclara BioSciences

Affymetrix
Agilent

\section{Althea Technologies}

Capital Genomix

Celera
Chromagen

Curagen

GeneFormatics

Genomic Solutions

Hybridon

Iconix Pharmaceuticals

Immusol

Incyte Genomics

IntegraGen

NimbleGen Systems

RNA-TEC

Sigma-Genosys

Xantos Biomedicine microarray scanners and readers instruments for genomics and proteomics microarray services detection and assay proteome interaction map for Drosophila

Genomics-based drug target discovery

Cyclicons synthetic fluorescent DNA probes for drug discovery

Custom oligonucleotide synthesis

Disease-specific and other cDNA libraries
LabCard microfluidics 'lab-on-a-chip' systems for biochemical and cellular analysis,

GeneChip microarray systems, including human whole-genome DNA microarray;

Workstation for RNA, DNA or protein analysis; human whole-genome DNA microarray;

PCR-based high-throughput gene-expression profiling

GeneSystem 320 differential gene-expression profiling; custom antibodies;

Discovery System web-based informatics tools for accessing the Celera annotated genomes databases; bioinformatics services; DNA sequencing; target validation Fluorescence based products, kits and services for gene expression and protein

GeneScape portal for genome analysis tools; proteomics research for drug target discovery;

Automated instrumentation for genomics and proteomics, software, consumables and services. Investigator automated system for proteomics

DrugMatrix databases and software platform for chemogenomics-based research

Ribozyme-based Inverse Genomics platform for identification of drug target genes

Annotated gene and expressed sequence tag databases; Proteome BioKnowledge Library species-specific protein information databases; bioinformatics software

GenomeHIP (Genome Hybrid Identity Profiling) gene-mapping technology; target discovery

Custom and off-the-shelf microarrays; human whole-genome DNA microarray

Tools for molecular biology; oligonucleotide libraries for gene expression profiling for

human, mouse, rat, Bacillus subtilis, zebrafish, Escherichia coli including multiplexed gene-expression analysis

Mountain View, California

Santa Clara, California

Palo Alto, California

San Diego, California

Chantilly, Virginia

Rockville, Maryland

San Diego, California

Branford, Connecticut

San Diego, California

Ann Arbor, Michigan

Cambridge, Massachusetts

Mountain View, California

San Diego, California

Palo Alto, California

Evry, France

Madison, Wisconsin

Leuven, Belgium

The Woodlands, Texas

Munich, Germany

Martinsried, Germany

The Woodlands, Texas

Custom transgenic mouse and rat models; drug discovery

Gene-knockout and gene-function databases; transgenic mice for target identification and validation

Custom transgenic and knockout mice for target identification and validation; cDNA

microarrays; SAGE analysis
Cologne, Germany www.aclara.com

www.affymetrix.com

www.agilent.com

www.altheatech.com

www.capitalgenomix.com

www.celera.com

www.chromagen.com

www.curagen.com

www.geneformatics.com

www.genomicsolutions.com

www.hybridon.com

www.iconixpharm.com

www.immusol.com

www.incyte.com

www.integragen.com

www.nimblegen.com

www.rna-tec.com

www.sigma-genosys.com

www.xantos.de ngenium Pharmaceuticals

Lexicon Genetics

memorec Stoffe www.ingenium-ag.com

www.lexgen.com 


$\begin{array}{llll}\text { Company } & \text { Products/activity } & \text { Location } & \text { URL } \\ \text { Nucleis } & \text { Custom transgenic mice } & \text { Lyon, France } & \text { www.nucleis.com } \\ \text { OzGene } & \text { Custom transgenic and knockout mice for target identification and validation } & \text { Canning Vale, Australia } & \text { www.ozgene.com } \\ \text { Zygogen } & \text { Transgenic zebrafish system for target identification and validation } & \text { Atlanta, Georgia } & \text { www.zygogen.com }\end{array}$

\section{Gene knockdown and RNA interference}

Ambion

atugen
Benitec
Cenix BioScience
Dharmacon
Eurogentec
GeneTools
Imgenex
InvivoGen
Mirus
MWG Biotech

\section{New England Biolabs}

Novagen

OligoEngine

Promega

\section{Proligo}

QIAGEN

Sequitur

Spring Bioscience

Xeragon
Silencer range of kits, vectors, siRNAs and reagents for RNAi; kits and products for RNA synthesis, isolation, quantitation and analysis siRNA-based drug discovery; custom phosphorothioate antisense RNAs and siRNAs for drug target identification and validation

Proprietary gene silencing RNAi technology

Genome-based high-throughput applications of RNA interference; siRNA design

siRNA kits and arrays; custom RNAs; large-scale RNA synthesis

Custom siRNA for RNAi

Morpholino antisense oligonucleotides

Plasmid-based GeneSuppressor siRNA kits for RNA interference

siRNA cloning vectors; custom siRNA in plasmid and viral vectors

Products for gene transfer and RNAi

Custom oligonucleotide synthesis; Dharmacon siRNA kits and arrays; custom sequencing; microarrays and oligo sets

HiScribe RNAi transcription kit

RiboJuice siRNA transfection reagent

pSUPER RNAi vector system for gene silencing; custom oligonucleotides for all applications

Vectors, reagents and kits for genomics, proteomics and cellular analysis; kits for siRNA production and delivery

Custom and speciality DNA and RNA oligos; siRNA oligos for RNAi

Custom siRNAs and transfection reagents; Cancer siRNA Oligo Set Version 1.0

Antisense and siRNA compounds

Knockdown kits for producing siRNAs from target DNA sequence; products for gene

cloning, target screening and protein-expression analysis

siRNA design tool; custom siRNA synthesis
Austin, Texas

Berlin, Germany

St Lucia, Queensland

Dresden, Germany

Lafayette, Colorado

Seraing, Belgium

Philomath, Oregon

San Diego, Califormia

San Diego, California

Madison, Wisconsin

Ebersberg, Germany

Beverly, Massachusetts

Madison, Wisconsin

Seattle, Washington

Madison, Wisconsin

Hamburg, Germany

Venlo, the Netherlands

Natick, Massachusetts

Fremont, California

Germantown, Maryland www.ambion.com

www.atugen.com

www.benitec.com.au

www.cenix-bioscience.com

www.dharmacon.com

www.eurogentec.be

www.gene-tools.com

www.imgenex.com

www.invivogen.com

www.genetransfer.com

www.mwg-biotech.com

www.neb.com

www.novagen.com

www.oligoengine.com

www.promega.com

www.proligo.com

www.qiagen.com

www.sequiturinc.com

www.springbio.com

www.xeragon.com

\section{General}

Amersham Biosciences

Apogent Technologies

Applied Biosystems

Aviva Biosciences

Axon Instruments

BD Biosciences

Beckman Coulter

Bio-Rad

Caliper Technologies

\section{Cellomics}

Chemicon

Cytomyx

Fluidigm

Gyros

Hamilton Company

LumiCyte

Luminex Corporation

Invitrogen

MDS Pharma

PerkinEImer Life Sciences

Protedyne

Roche Diagnostics

Tecan

Stratagene

- see advertisement
Instruments, equipment and products for genomics, proteomics and cellular assays; workstations and reagents for DNA microarrays; IN Cell Analyser confocal imaging system for rapid cellular assays

Labware and equipment for the life sciences

DNA sequencers, protein sequencers, mass spectrometers, chromatography systems,

PCR systems, peptide synthesizers, nucleic acid synthesizers

SEAL Microchips for high-throughput patch-clamp ion-channel measurements

Scanners and microarray software; ImageExpress for live-cell assays

Culture media, radioassay kits, FACS range of flow cytometers; monoclonal antibodies, antibody arrays for proteomics; reagents and biochemicals for molecular biology

Automated tools for molecular biology, biochemistry, genomics and proteomics

Products, instruments and software for life-sciences research; Bio-Plex system for multiplex assays

LabChip 'lab-on-a-chip' microfluidic systems for high-throughput screening for drug discovery, biological and genetic research

ArrayScan HCS and KineticScan HCS systems for automated cell-based high-content screening for drug discovery

Off-the shelf and custom antibodies; reagents and kits for bioochemistry and molecular biology

Antibodies, cDNAs and cell lines for molecular and cell biology; services for genomics and proteomics, cell-line production

Microfluidics chip for protein crystallization

CD microlaboratory for sample preparation for protein mass spectrometry

Microlab automated liquid-handling workstations

Protein biomarker profiles for drug development

XMAP platform for microbead-based multiplex assays

Kits and reagents for genomics, proteomics, molecular and cell biology; Lipofectamine 2000 for siRNA transfection

Services company for all aspects of drug discovery

Automated systems for liquid handling and sample preparation; high-throughput human DNA microarrrays; HydroGel BioChip

BioCube automated systems for research and genome-based drug discovery

Reagents and kits for molecular biology, functional genomics and proteomics research

Robotic laboratory workstations and automated solutions for genomics and proteomics

Tools and reagents for molecular biology, genomics, proteomics, drug discovery and

toxicology; GeneEraser siRNA transfection reagents
Piscataway, New Jersey

www.amershambiosciences.com

Portsmouth, New Hampshire www.apogent.com

Foster City, California

home.appliedbiosystems.com

San Diego, California

Foster City, California

Franklin Lakes, New Jersey

www.avivabio.com

www.axon.com

www.bd.com

Fullerton, California

www.beckmancoulter.com

Hercules, California

www.bio-rad.com

Mountain View, California

www.calipertech.com

Pittsburgh, Pennsylvania

www.cellomics.com

Ternecula, California

www.chemicon.com

Cambridge, UK

www.cytomyx.com

San Francisco, California

Uppsala, Sweden

Reno, Nevada

Fremont, California

Austin, Texas

Carlsbad, California

Lincoln, Nebraska

Boston, Massachusetts

www.fluidigm.com

www.gyros.com

hamiltoncomp.com

www.lumicyte.com

www.luminexcorp.com

www.invitrogen.com

www.mdsps.com

lifesciences.perkinelmer.com

Windsor, Connecticut

Lewes, UK

Medford, Massachusetts

La Jolla, California

www.protedyne.com

www.roche-applied-science.com

www.tecan.com

www.stratagene.com 